PHYSICAL REVIEW B 79, 134416 (2009)

S

\title{
Evidence of spin singlet ground state in the frustrated antiferromagnetic ring $\mathrm{Cr}_{8} \mathrm{Ni}$
}

\author{
Yuji Furukawa \\ Department of Physics and Astronomy and Ames Laboratory, Iowa State University, Ames, Iowa 50011, USA \\ Kazuki Kiuchi and Ken-ichi Kumagai \\ Division of Physics, Graduate School of Science, Hokkaido University, Sapporo 060-0810, Japan \\ Yoshitami Ajiro \\ Department of Chemistry, Kyoto University, Kyoto 606-8502, Japan and CREST, Japan Science and Technology (JST), \\ Tokyo 102-8581, Japan \\ Yasuo Narumi \\ Institute of Material Research, Tohoku University, Sendai 980-8577, Japan \\ Masahiro Iwaki and Koichi Kindo \\ The Institute for Solid State Physics, The University of Tokyo, Kashiwa 277-8581, Japan
}

Alberto Bianchi, Stefano Carretta, and Paolo Santini

Dipartimento de Physica, Università di Parma, I-43100 Parma, Italy

and $S^{3}$-CNR-INFM, I-41100 Modena, Italy

Ferdinando Borsa

Department of Physics and Astronomy and Ames Laboratory, Iowa State University, Ames, Iowa 50011, USA and Department of Physics, "A. Volta” and Unita' CNISM, Universita' di Pavia, I-27100 Pavia, Italy

Grigore A. Timco and Richard E. P. Winpenny

School of Chemistry, University of Manchester, Oxford Road, Manchester M13 9PL, United Kingdom

(Received 27 January 2009; published 13 April 2009)

\begin{abstract}
The magnetic properties of an odd spin number antiferromagnetic ring $\mathrm{Cr}_{8} \mathrm{Ni}$ have been investigated by high-field magnetization, specific-heat, and nuclear-magnetic-resonance measurements at very low temperatures (below $1 \mathrm{~K}$ ). It is found that the ground state of this frustrated spin ring is a singlet state with total spin value $S_{\mathrm{T}}=0$ and zero expectation value of the local spins. The energy-level structure for low-lying excited total spin state $S_{\mathrm{T}} \neq 0$ shows a breakdown of the Landé interval rule in agreement with theoretical results. Evidences of sizeable Dzyaloshinski-Moriya interactions have been found in the magnetic-field dependence of lowtemperature specific-heat and magnetization data.
\end{abstract}

DOI: 10.1103/PhysRevB.79.134416

PACS number(s): 75.50.Xx, 75.75.+a, 76.60.-k

\section{INTRODUCTION}

Recently there has been intense experimental and theoretical effort in synthesis and investigation of nanoscale molecular magnetic systems, which are composed of a controllable number of transition-metal ions. ${ }^{1}$ A shell of organic ligands shields the individual molecular magnets from each other so that the magnetic interaction between the neighboring molecular magnets is very small and the observed magnetic properties of the bulk samples are considered to originate from intramolecular magnetic interactions only. The discovery of quantum-phenomena-like quantum tunneling of the magnetization (QTM) observed in single molecule magnets (SMM) such as $\mathrm{Mn}_{12}$-ac (Ref. 2) and $\mathrm{Fe}_{8}$ (Ref. 3) has further increased the interest in the investigation of nanoscale molecular magnets. Among nanoscale molecular magnets are antiferromagnetic (AF) rings that have an almost coplanar ring shape with a number $(N)$ of transition-metal ions. Even-number AF rings with $N=6,8,10,12$, and 18 are relatively common for transition-metal ions with different spins such as $\mathrm{Fe}^{3+}(s=5 / 2),{ }^{4-7} \mathrm{Cr}^{3+}(s=3 / 2),{ }^{8} \mathrm{~V}^{3+}(s=1),{ }^{9}$ and $\mathrm{Cu}^{2+}(s=1 / 2) .{ }^{10} \mathrm{~A}$ common feature of all even-number rings is to have a spin singlet $S_{\mathrm{T}}=0$ ground state due to the dominant antiferromagnetic Heisenberg exchange couplings between nearest-neighbor spins. Due to the finite-size effects, AF rings have a discrete energy spectrum and the lowest-lying excited states for the total spin $S_{\mathrm{T}}$ is known to be approximately given by so-called Landé interval rule $E\left(S_{\mathrm{T}}\right)=(2 J / N) S_{\mathrm{T}}\left(S_{\mathrm{T}}+1\right)$, where $J$ is the antiferromagnetic exchange coupling constant. ${ }^{11}$

In the case of odd-number AF rings, magnetic properties are expected to be changed drastically due to spin frustration. The simplest systems of odd-number molecular AF rings are the $s=1 / 2$ three spin system with an antiferromagnetic interaction known as $\mathrm{V}_{15}$ (Ref. 12) and $\mathrm{Cu}_{3} .{ }^{13}$ In these frustrated triangular AF spin systems, the ground state is a magnetic $S_{\mathrm{T}}=1 / 2$ state. ${ }^{12}$ Odd number AF rings with $N>3$ have been more difficult to synthesize. Quite recently a breakthrough in 

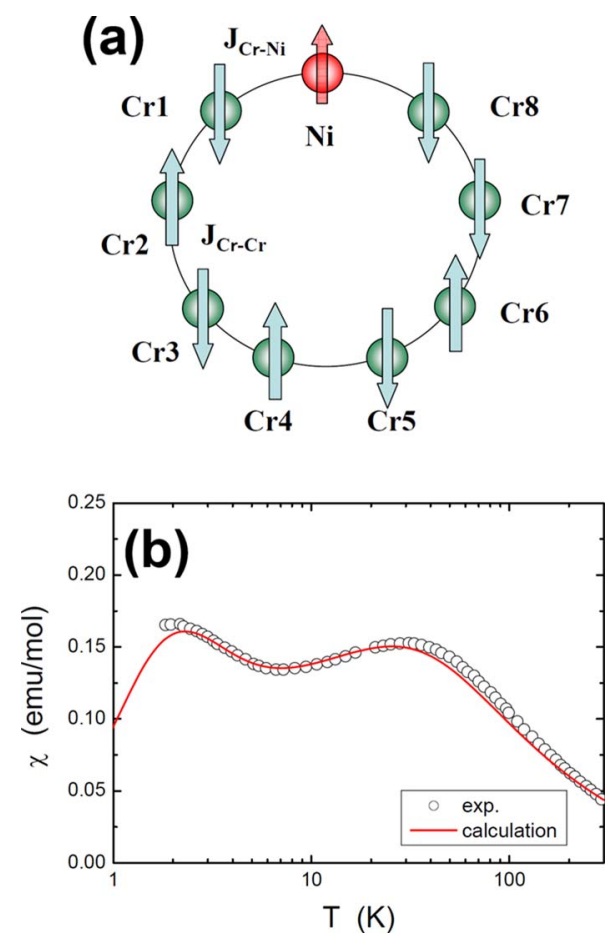

FIG. 1. (Color online) (a) Schematic view of $\mathrm{Cr}_{8} \mathrm{Ni}$. (b) Temperature dependence of magnetic susceptibility of $\mathrm{Cr}_{8} \mathrm{Ni}$ from Ref. 14. The solid line shows the theoretical calculated results from Eqs. (1) and (4).

the synthesis of a new family of odd-number AF rings with $N=9$ was achieved. ${ }^{14-16}$ A heterometallic $\mathrm{Cr}$-based ringshaped AF magnet $\left(\mathrm{C}_{2} \mathrm{H}_{11}\right)_{2} \mathrm{NH}_{2}\left\{\mathrm{Cr}_{8} \mathrm{NiF}_{9}\left[\mathrm{O}_{2} \mathrm{CC}\left(\mathrm{CH}_{3}\right)_{3}\right]_{18}\right\}$ (abbreviated as, $\mathrm{Cr}_{8} \mathrm{Ni}$ ) was synthesized by Winpenny and co-workers. ${ }^{14,15}$ As a matter of fact $\mathrm{Cr}_{8} \mathrm{Ni}$ is the first oddnumber AF ring with $N>3$. This system has spin frustration effects because all antiferromagnetic interactions cannot be simultaneously satisfied. In this respect, this system can be regarded as a magnetic analog of the Möbius strip. ${ }^{14}$ Since for an odd number of spins with AF interactions it is impossible to have antiparallel alignment for all nearest-neighbor spins, the region where the spins are not antiparallel to each other can be considered as a knot of the Möbius strip.

The precursor of $\mathrm{Cr}_{8} \mathrm{Ni}$ is an even-number $\mathrm{AF}$ ring $\left\{\mathrm{Cr}_{8} \mathrm{~F}_{8}\left[\mathrm{O}_{2} \mathrm{CC}\left(\mathrm{CH}_{3}\right)_{3}\right]_{16}\right\} 0.25 \mathrm{C}_{6} \mathrm{H}_{14}$ (in short, $\mathrm{Cr}_{8}$ ) whose ground state is a spin singlet $S_{\mathrm{T}}=0$ state due to AF interaction $\left(J_{\mathrm{Cr}-\mathrm{Cr}} \sim 16.9 \mathrm{~K}\right)$ between nearest-neighbor $\mathrm{Cr}^{3+}$ $(s=3 / 2)$ spins. ${ }^{17}$ The $\mathrm{Cr}_{8} \mathrm{Ni}$ is basically obtained by the insertion of a Ni${ }^{2+}(s=1)$ ion into the $\mathrm{Cr}_{8}$ ring. The schematic structure of the core of $\mathrm{Cr}_{8} \mathrm{Ni}$ is shown in Fig. 1(a). The metal ions are bridged by one fluorine ion and two $\left(\mathrm{CH}_{3}\right)_{3} \mathrm{CCO}_{2}$ radicals. ${ }^{14}$ The antiferromagnetic interactions between the $\mathrm{Cr}$ ions, and between the $\mathrm{Cr}$ and the $\mathrm{Ni}$ ions are reported to be $J_{\mathrm{Cr}-\mathrm{Cr}}=16 \mathrm{~K}$ and $J_{\mathrm{Cr}-\mathrm{Ni}}=70 \mathrm{~K}$, respectively, from the fit of the temperature dependence of magnetic susceptibility $\chi$ which shows peaks around 2 and $25 \mathrm{~K}$ [see Fig. 1(b) $].{ }^{14}$ From the fitting of the magnetic susceptibility in the temperature range $T=1.6-300 \mathrm{~K}$, it is also inferred that the ground state is a singlet $S_{\mathrm{T}}=0$ and that the first-excited state is a $S_{\mathrm{T}}=1$ state at $3.7 \mathrm{~K}$ from the ground state. The fit was based on a spin Hamiltonian that included Heisenberg ex- change terms only. ${ }^{14}$ The conclusion about the singlet ground state in this frustrated ring is interesting and it thus requires more direct experimental evidence. In order to elucidate the ground state of the $\mathrm{Cr}_{8} \mathrm{Ni}$ system from experimental point of view, it is important to investigate the magnetic properties at much lower temperature, i.e., well below the lowest energy gap. In this paper, we have carried out high-field magnetization, specific-heat, and nuclear-magnetic-resonance (NMR) measurements at very low temperatures down to $0.1 \mathrm{~K}$. Our experimental results clearly show an evidence of a spin singlet ground state for the $\mathrm{Cr}_{8} \mathrm{Ni}$ system and allow us to establish the energy separation of the low-lying quantum magnetic states. The experimental results are in good agreement with predictions from theoretical calculations. Interestingly, the width of the steps in the field dependence of the magnetization and the lack of sharp minima in the field dependence of the specific heat show that sizeable Dzyaloshinski-Moriya (DM) interactions are present, and lead to anticrossings (ACs) between states of different total spin.

\section{EXPERIMENTAL}

Polycrystalline samples of $\left(\mathrm{C}_{2} \mathrm{H}_{11}\right)_{2} \mathrm{NH}_{2}\left[\mathrm{Cr}_{8} \mathrm{NiF}_{9}\right.$ $\left.\left(\mathrm{O}_{2} \mathrm{CC}\left(\mathrm{CH}_{3}\right)_{3}\right)_{18}\right]$ were prepared as described in Ref. 14. The magnetization curve was measured using a pulsed magnet up to $55 \mathrm{~T}$ at $T=0.1 \mathrm{~K}$ utilizing a ${ }^{3} \mathrm{He}-{ }^{4} \mathrm{He}$ dilution refrigerator at the Institute for Solid State Physics (ISSP) of the University of Tokyo. The duration of the pulse is about $5 \mathrm{~ms}$. The specific heat was measured by the relaxation method with two time-constant data fitting utilizing a Quantum design physical property measurement system (PPMS-14T) with a ${ }^{3} \mathrm{He}$ cryostat at the ISSP. The ${ }^{1} \mathrm{H}$ NMR measurement was carried out utilizing a homemade phase-coherent spin-echo pulse spectrometer down to $0.05 \mathrm{~K}$ using a dilution refrigerator at Hokkaido University. The ${ }^{1} \mathrm{H}$ NMR spectra were obtained from Fourier transform (FT) of the half-echo signal at a fixed magnetic field.

\section{EXPERIMENTAL RESULTS}

Figure 2(a) shows the magnetization $(M)$ curves for $\mathrm{Cr}_{8} \mathrm{Ni}$ at $T=0.1 \mathrm{~K}$ for increasing magnetic fields, where a clear stepwise increase in magnetization is observed. The magnetization curves are nearly the same with no sizeable hysteresis for the up and down magnetization processes at this temperature. The magnetization rapidly increases step by step with plateaus at $\sim 2 \mu_{B}, \sim 4 \mu_{B}, \sim 6 \mu_{B}, \sim 8 \mu_{B}$, and $\sim 10 \mu_{B}$ at the transition fields $H_{n}(n=1,2 \ldots)$. The level crossing (LC) fields $H_{n}$ are determined by the peak positions of the $d M / d H$ curves [see, Fig. 2(b)] and are found to be 2.81, 12.8, 18.3, 23.5, and 28.5 T, for $n=1,2,3,4$, and 5, respectively. The linewidth $(\delta H)$ measured at half amplitude for each peak in $d M / d H$ is estimated to be $3.07,1.61,1.69$, 2.15 , and $2.06 \mathrm{~T}$ for $n=1,2,3,4$, and 5, respectively. These values are much higher than the expected thermal width 0.26 $\mathrm{T}$ for $T=0.1 \mathrm{~K}$ estimated from the equation $\delta H$ $=3.53 k_{B} T / g \mu_{B}$ with the assumption of $g \sim 2$. As will be discussed below, the broadening is also too large to be attributed merely to the distribution of level crossing fields $H_{n}$ in 

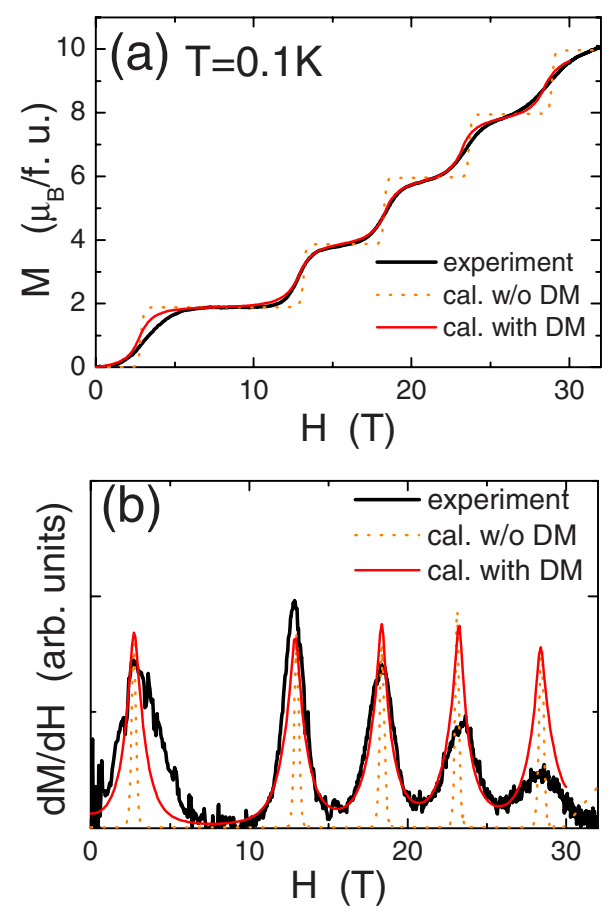

FIG. 2. (Color online) (a) Magnetization curve of $\mathrm{Cr}_{8} \mathrm{Ni}$ measured at $T=0.1 \mathrm{~K}$ (black solid line). Red lines show the theoretical calculated results from Eq. (1) for $T=0.1 \mathrm{~K}$ with a set of parameter of $J_{\mathrm{Cr}-\mathrm{Cr}}=14.7 \mathrm{~K}, J_{\mathrm{Cr}-\mathrm{Ni}}=85 \mathrm{~K}, d_{\mathrm{Cr}}=-0.42 \mathrm{~K}$, and $d_{\mathrm{Ni}}=-4.9 \mathrm{~K}$ (dotted line; without DM interaction, solid line; with DM interaction). (b) $d M / d H$ curve at $T=0.1 \mathrm{~K}$. Red lines show the theoretical calculated results for $T=0.1 \mathrm{~K}$ (dotted line; without DM interaction, solid line; with DM interaction).

the powder sample and is thus an indication of the presence of level anticrossing effects.

If the ground state of the system were magnetic, one should observe a Brillouin function like increase for the initial magnetization. The observed convex downward initial slope clearly proves a nonmagnetic ground state with an energy gap to the first-excited state. Thus the $H_{n}$ can be attributed to the ground-state level crossing field from $S_{\mathrm{T}}=0$ to $S_{\mathrm{T}}=1$ for $n=1$, from $S_{\mathrm{T}}=1$ to $S_{\mathrm{T}}=2$ for $n=2$, and so on. The separations in magnetic field between the level crossing fields defined here as $\Delta H_{n}=H_{n}-H_{n-1}$ (where $H_{0}$ is zero) are shown in Fig. 3 as a function of $n$. The $n$ dependence of $H_{n}$

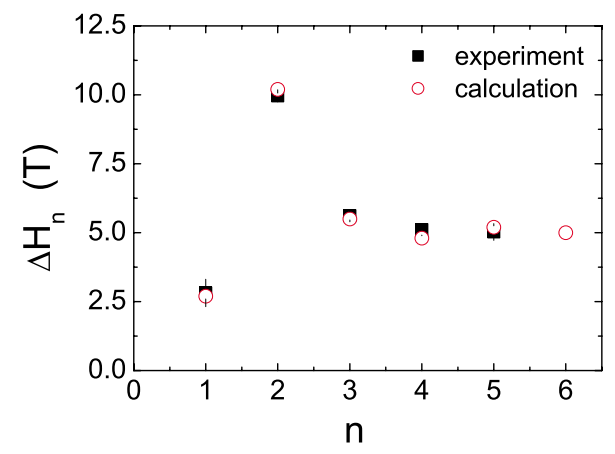

FIG. 3. (Color online) $H$ dependence of $\Delta H_{n}$. Closed and open symbols show experimental and theoretical results, respectively.

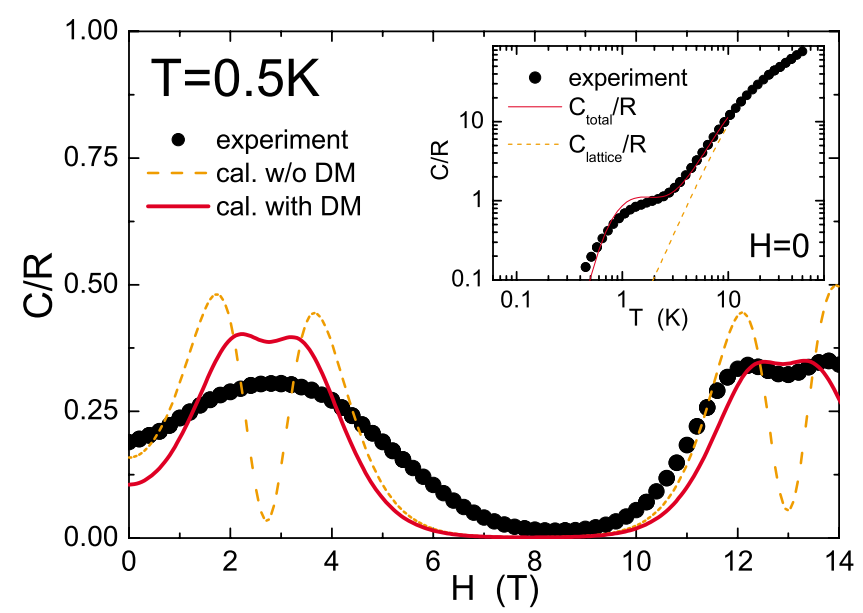

FIG. 4. (Color online) $H$ dependence of specific heat at $T$ $=0.5 \mathrm{~K}$. Red lines are theoretical calculated results for $T=0.5 \mathrm{~K}$ (broken line; without DM interaction, solid line; with DM interaction). The inset shows temperature dependence of specific heat measured under zero magnetic field. Red solid line in the inset shows calculated $C_{\text {total }}$. Dotted line shows $C_{\text {lattice }}$ calculated from the Eq. (3) with a set of parameter of $r=335, \Theta_{D}=180 \mathrm{~K}$, and $\delta$ $=0.27$.

for $\mathrm{Cr}_{8} \mathrm{Ni}$ does not follow the Landé interval rule, which predicts a separation $\Delta H$ independent of $n$.

Figure 4 shows the $H$ dependence of the specific heat $C_{\text {total }}$ at $T=0.5 \mathrm{~K}$ while the inset shows the temperature dependence of specific heat $C_{\text {total }}$ under zero magnetic fields. The measured specific heat is the sum of a magnetic contribution and a lattice contribution, i.e., $C_{\text {total }}=C_{\text {mag }}+C_{\text {lattice }}$. However, as we will discuss later, the observed specific heat at $T=0.5 \mathrm{~K}$ can be considered due mainly to the magnetic contributions $C_{\text {mag. }}$. A single broad peak is observed around the first level crossing $H_{1}=2.82 \mathrm{~T}$. At the second level crossing, one can resolve two peaks at 12.1 and $13.7 \mathrm{~T}$ with a small dip around 13.0 T. In presence of a level crossing one should indeed observe two peaks with a dip in the middle. This is due to a Schottky-type anomaly as it was observed in even-number AF rings $\mathrm{Fe}_{6}$ (Ref. 18) and $\mathrm{Cr}_{8} \cdot{ }^{19}$ The two-level Schottky model predicts $C_{s}=\left[\Delta(H) / k_{B} T\right]^{2} \exp$ $\left[\Delta(H) / k_{B} T\right] /\left\{1+\exp \left[\Delta(H) / k_{B} T\right]\right\}$, a behavior which yields a maximum when $\Delta \sim 2.5 k_{B} T$. Near level crossing field, the energy separation $\Delta(H)$ between two lowest-lying states can be expressed approximately as $\Delta(H) \sim g \mu_{B}\left|H_{n}-H\right|$ and thus two maxima are expected at $H \sim H_{n} \pm 2.5 k_{B} T / g \mu_{B}$ with a dip at $H_{n}$ where $\Delta(H)$ is smallest. In presence of a pure level crossing the dip between the two Schottky-type peaks should go down to zero since at level crossing $\Delta(H)=0$ while in presence of a level repulsion (level anticrossing) the dip can be much less pronounced and it gives a measure of the gap at the anticrossing. ${ }^{18}$ As shown in Fig. 4 a small dip superimposed on a broad maximum is observed only at the second level crossing. The broadening of the peaks cannot be ascribed to the distribution of the level crossing fields in a powder sample as will be demonstrated below in the discussion of the broadening of the magnetization steps. Thus one can conclude that, at both the first and second level crossings, a sizeable gap exists between the two crossing levels (level anticrossing). 


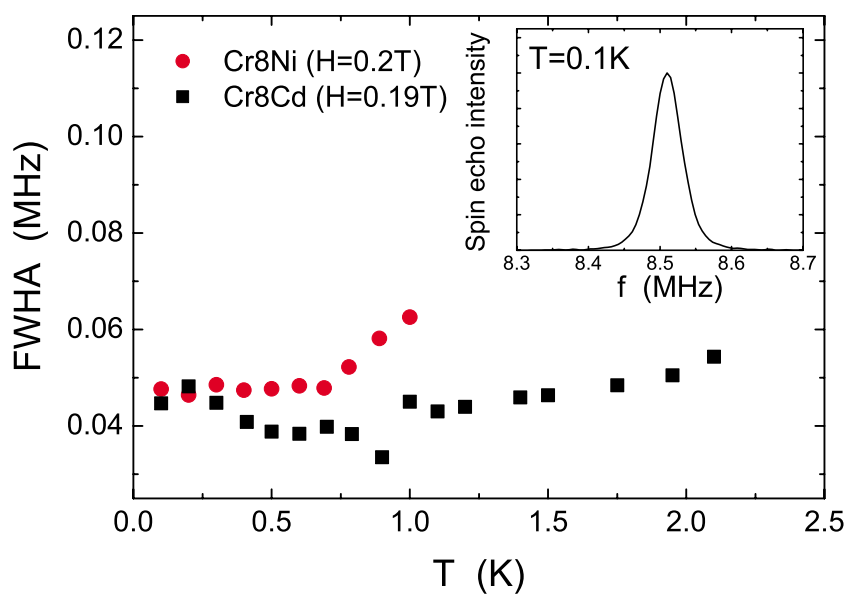

FIG. 5. (Color online) Temperature dependence of FWHA of ${ }^{1} \mathrm{H}-\mathrm{NMR}$ spectra in $\mathrm{Cr}_{8} \mathrm{Ni}$ (closed circles) at $H=0.2 \mathrm{~T}$ and $\mathrm{Cr}_{8} \mathrm{Cd}$ (closed squares) at $H=0.19 \mathrm{~T}$. The inset shows a typical ${ }^{1} \mathrm{H}-\mathrm{NMR}$ spectrum in $\mathrm{Cr}_{8} \mathrm{Ni}$ at $T=0.1 \mathrm{~K}$.

The spin singlet ground state in $\mathrm{Cr}_{8} \mathrm{Ni}$ is also revealed by proton NMR spectrum measurements at $H=0.2 \mathrm{~T}$. We observed a single narrow line of ${ }^{1} \mathrm{H}-\mathrm{NMR}$ as shown in the inset of Fig. 5. The full width at half amplitude (FWHA) decreases from $\sim 62 \mathrm{kHz}(\sim 15 \mathrm{Oe})$ at $T=1 \mathrm{~K}$ on lowering temperature and becomes almost independent of $T[\sim 42 \mathrm{kHz}$ $(\sim 10 \mathrm{Oe})]$ below $\sim 0.6 \mathrm{~K}$ as shown in Fig. 5 . The FWHA $\sim 10$ Oe can be explained by nuclear-nuclear dipolar interactions. In fact the nuclear dipolar field between two protons is of the order of $\gamma_{\mathrm{N}} \hbar / r^{3} \sim 8.3$ Oe when one assumes an internuclear distance $r=1.5 \AA\left(\gamma_{\mathrm{N}}\right.$ is the gyromagnetic ratio of a proton). Thus we may conclude there is no magnetic broadening in the spectrum due to the $\mathrm{Cr}$ and/or $\mathrm{Ni}$ spin moments. This is consistent with a total spin $S_{\mathrm{T}}=0$ ground state and also with a zero expectation value for the local spin component at each ion site. To check this conclusion, we have measured the FWHA of ${ }^{1} \mathrm{H}-\mathrm{NMR}$ spectrum at nearly the same magnetic field $H=0.19 \mathrm{~T}$ in a similar odd-number Cr-based AF ring $\left(\mathrm{Me}_{2} \mathrm{CH}\right)_{2} \mathrm{NH}_{2}\left\{\mathrm{Cr}_{8} \mathrm{CdF}_{9}\left[\mathrm{O}_{2} \mathrm{CC}\left(\mathrm{CH}_{3}\right)_{3}\right]_{18}\right\}$ (in short, $\mathrm{Cr}_{8} \mathrm{Cd}$ ) which has a spin singlet ground state with a first-excited spin triplet state at about $3 \mathrm{~K}$ above the ground state. ${ }^{20,21}$ As shown in Fig. 5, the FWHA for both compounds coincide at low temperatures within our experimental uncertainty, indicating that the FWHA of about 10 Oe originates from the nuclear dipolar interaction, which is the same for isostructural $\mathrm{Cr}_{8} \mathrm{Ni}$ and $\mathrm{Cr}_{8} \mathrm{Cd}$.

\section{THEORETICAL ANALYSIS}

We turn now to a quantitative comparison of the experimental results with the theoretical calculations. The starting spin Hamiltonian for the odd-number AF ring is

$$
\begin{aligned}
\mathcal{H}= & J_{\mathrm{Cr}-\mathrm{Ni}}\left(\mathbf{s}_{1} \cdot \mathbf{s}_{\mathrm{Ni}}+\mathbf{s}_{8} \cdot \mathbf{s}_{\mathrm{Ni}}\right)+\sum_{i=1}^{7} J_{\mathrm{Cr}-\mathrm{Cr}} \mathbf{s}_{i} \cdot \mathbf{s}_{i+1} \\
& +\sum_{i=1}^{8} d_{\mathrm{Cr}}\left(s_{z}^{2}(i)-\frac{1}{3} s_{\mathrm{Cr}}\left(s_{\mathrm{Cr}}+1\right)\right) \\
& +d_{\mathrm{Ni}}\left(s_{z, \mathrm{Ni}}^{2}-\frac{1}{3} s_{\mathrm{Ni}}\left(s_{\mathrm{Ni}}+1\right)\right)
\end{aligned}
$$
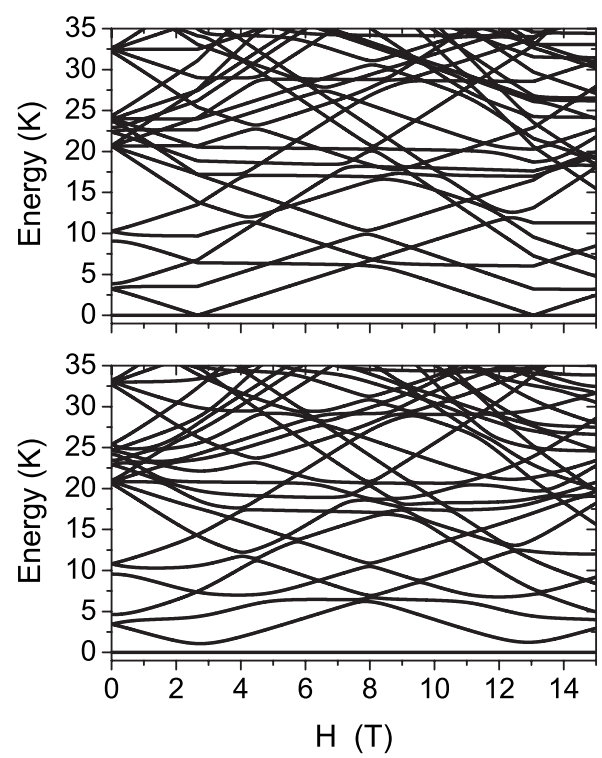

FIG. 6. Magnetic-field dependence of the low-lying energy levels of $\mathrm{Cr}_{8} \mathrm{Ni}$ without (top) or with (bottom) DM interactions described by Eq. (4). The fields make an angle of $45^{\circ}$ with the $z$ axis. For each value of $H$ the ground-state energy has been set to zero.

$$
-g_{\mathrm{Cr}} \mu_{B} \sum_{i=1}^{8} \mathbf{H} \cdot \mathbf{s}_{i}-g_{\mathrm{Ni}} \mu_{B} \mathbf{H} \cdot \mathbf{s}_{\mathrm{Ni}}
$$

where $i$ labels the eight $\mathrm{Cr}$ ions (spin). The first and second terms describe the dominant isotropic Heisenberg exchange interaction. We assume two different exchange constants: one between $\mathrm{Cr}$ and $\mathrm{Ni}$ spins $\left(J_{\mathrm{Cr}-\mathrm{Ni}}\right)$, and another one between $\mathrm{Cr}$ spins $\left(J_{\mathrm{Cr}-\mathrm{Cr}}\right)$. The third and fourth terms describe local crystal fields $\left(d_{\mathrm{Cr}}\right.$ and $d_{\mathrm{Ni}}$ represent uniaxial anisotropy constants for $\mathrm{Cr}$ and $\mathrm{Ni}$ ions, respectively), and the fifth and sixth terms are the Zeeman interactions. The two exchange parameters have been determined to be $J_{\mathrm{Cr}-\mathrm{Cr}}=14.7 \mathrm{~K}$ and $J_{\mathrm{Cr}-\mathrm{Ni}}=85 \mathrm{~K}$ by fitting magnetization and susceptibility data. Reasonable values of $d_{\mathrm{Cr}}$ and $d_{\mathrm{Ni}}$ turn out to produce tiny effects in the powder properties. Hence we assume $d_{\mathrm{Cr}}$ $=-0.42 \mathrm{~K}$ as in the parent $\mathrm{Cr}_{8} \mathrm{Zn}$ compound ${ }^{22}$ and the same ratio of $d_{\mathrm{Cr}}$ to $d_{\mathrm{Ni}}$ as in $\mathrm{Cr}_{7} \mathrm{Ni}^{23}$ yielding $d_{\mathrm{Ni}}=-4.9 \mathrm{~K}$.

The Hamiltonian is diagonalized by following the procedure described in Ref. 17. The calculated eigenstates and eigenvalues have been used to evaluate the $H$ dependence of the magnetization and of the magnetic specific heat $\left(C_{\text {mag }}\right)$, as well as the $T$ dependence of $\chi$ and $C_{\text {mag. }}$. Since we used polycrystalline samples, all calculated results are powder averaged where molecules are assumed to be randomly oriented. Therefore the calculated results can be compared directly with the experimental observations. By using $g_{\mathrm{Cr}}$ $=1.98$ and $g_{\mathrm{Ni}}=2.2$, the $T$ dependence of $\chi$ [see the solid line in the Fig. 1(b)] and the position of the observed level crossing fields $H_{n}$ are well reproduced [see Fig. 2(b)]. The $n$ dependence of $H_{n}$ is also well reproduced by the theory as shown in Fig. 3. On the other hand, the width of the calculated peaks in the $d M / d H$ curve is much smaller than the 
experimental results as shown in Fig. 2(b). The origin of the broadening of the magnetization steps is likely to be the same as the origin of the broadening of the specific-heat peaks and is discussed after the presentation of the specific- heat results.

The temperature dependence of the calculated specific heat $C_{\text {total }}\left(=C_{\text {mag }}+C_{\text {lattice }}\right)$ is shown by a red solid line in the inset of Fig. 4. $C_{\text {mag }}$ was calculated from the equation ${ }^{20}$

$$
\frac{C_{\mathrm{mag}}}{R}=\left(k_{B} T\right)^{-2}\left[\frac{\sum_{i} \varepsilon_{i}^{2} \exp \left(-\frac{\varepsilon_{i}}{k_{B} T}\right) \sum_{i} \exp \left(-\frac{\varepsilon_{i}}{k_{B} T}\right)-\left[\sum_{i} \varepsilon_{i} \exp \left(-\frac{\varepsilon_{i}}{k_{B} T}\right)^{2}\right]}{\left[\sum_{i} \exp \left(-\frac{\varepsilon_{i}}{k_{B} T}\right)\right]^{2}}\right],
$$

with eigenvalues $\varepsilon_{i}$ obtained from Eq. (1). $R$ is the gas constant with $8.3141 \mathrm{~J} \mathrm{~mol}^{-1} \mathrm{~K}^{-1}$. The lattice contribution to $C_{\text {total }}$ has been estimated from a phenomenological mode ${ }^{24}$

$$
\frac{C_{\text {lattice }}}{R}=\frac{234 r T^{3}}{\left(\Theta_{D}+\delta T^{2}\right)^{3}},
$$

where $r$ is the number of atoms per molecule, and $\Theta_{D}$ and $\delta$ are parameters. For $\mathrm{Cr}_{8} \mathrm{Ni}$, we used $r=335, \Theta_{D}=180 \mathrm{~K}$, and $\delta=0.27$ which are very close to those reported for the isostructural AF ring $\mathrm{Cr}_{8} \mathrm{Cd} .{ }^{20}$ The broken line in the inset of Fig. 4 shows the calculated $C_{\text {lattice }}$. Note that the specific heat at low temperatures (below $1 \mathrm{~K}$ ) is dominated by the magnetic contribution $\left(C_{\text {mag }}\right)$. The calculated $C_{\text {total }}$, resulting from the sum of $C_{\text {mag }}$ and $C_{\text {lattice, }}$ is in good agreement with the experimental data (inset of Fig. 4). It should be noted that we did not use any scaling factors to fit the experimental data by the calculation. On the other hand the calculated $C_{\text {mag }}$ at $T$ $=0.5 \mathrm{~K}$ as a function of $H$, shown by a broken line in Fig. 4, is not in good agreement with the experiments. The problem is the remarkable broadening of the peaks in the experimental specific heat at level crossings, which should have the same origin as the broadening in the magnetization steps (see Fig. 2) and is not reproduced by calculations based only on Eq. (1). We have already introduced reasonable single-ion terms in the calculations; hence the broadening effects in the experiment cannot be simply attributed to a distribution of $H_{n}$ due to the random direction of crystal-field anisotropy axes in a powder sample. In principle, a distribution of exchange parameters from molecule to molecule resulting from disorder ( $J$ strain) might produce a broadening. For instance, in $\mathrm{Cr}_{7} \mathrm{Ni}$, a distribution with standard deviation of the order of $2.5 \%$ was found ${ }^{25}$ However we find that an unrealistically large value for the standard deviation has to be assumed to account for the entity of the observed effects. In addition, the effect of $J$ strain on the minimum in $C_{\text {mag }}$ at $2.7 \mathrm{~T}$ is much smaller than that on the minimum at $13 \mathrm{~T}$, whereas in experimental data the broadening is of the same entity in the two minima.

On the other hand, if the LCs are not true crossings but level anticrossings, $C_{\text {mag }}$ does not vanish at $H_{n}$ because the energy separation between two lowest-lying states, $\Delta(H)$, remains finite. By considering only the lowest multiplet for each value of $S$, even- $S$ and odd- $S$ multiplets belong to different irreducible representations of the ideal spin permutational symmetry of Eq. (1). Therefore crossings can be turned into anticrossings only by including in the Hamiltonian terms which break this symmetry, for instance, sitedependent Cr crystal fields. However, this kind of symmetry lowering would not significantly affect the first crossing at 2.7 T. In fact, only a DM interaction can directly mix the $S$ $=0$ and $S=1$ multiplets because it is the only term (beside the Zeeman term which does not break spin permutational symmetry) which is described by rank-one complex tensor operators $T_{Q}^{K}$ and because if $K>1\left\langle S=0\left|T_{Q}^{K}\right| S=1\right\rangle=0$. Hence, the absence of a minimum in $C_{\text {mag }}$ at $2.7 \mathrm{~T}$ provides strong evidence of the presence of DM interactions in the microscopic Hamiltonian. The most likely sources of this interaction are the two $\mathrm{Cr}-\mathrm{Ni}$ bonds because the inversion symmetry is maximally broken and the exchange interaction is the strongest. In addition, the orbital degrees of freedom which are behind the DM interaction are much less quenched in $\mathrm{Ni}^{2+}$ than in $\mathrm{Cr}^{3+}$ ions. To investigate the effect of DM interactions, we have made calculations assuming the simplest possible choice for their form:

$$
\mathcal{H}_{\mathrm{DM}}=\mathbf{D} \cdot\left(\mathbf{s}_{8} \times \mathbf{s}_{\mathrm{Ni}}\right)+\mathbf{D} \cdot\left(\mathbf{s}_{\mathrm{Ni}} \times \mathbf{s}_{1}\right),
$$

where we assume the two DM vectors to be equal and parallel to the $z$ axis. The actual DM vectors are very likely to be different than in Eq. (4) but our aim is not to look for a best fit of the data but merely to assess whether or not the DM interaction is a realistic mechanism to explain the observed behavior. We assume for the modulus of $\mathbf{D}$ the typical value $D=0.1 J_{\mathrm{Cr}-\mathrm{Ni}}$. The resulting spectrum is compared with that obtained for $D=0$ in Fig. 6. Sizeable anticrossings open up at $H_{n}$ with an amplitude depending on the angle between the applied field and the $z$ axis. The effect on $C_{\text {mag }}$ is shown by the red solid line in Fig. 4. The DM interactions remove the sharp minima at $H_{n}$ in agreement with the experimental results. In addition, the width of steps in the calculated field dependence of $M$ is in very good agreement with experimental data as shown by the red solid line in Figs. 2(a) and 2(b).

Having established the main interactions appearing in the microscopic Hamiltonian, we can study the properties of the resulting ground state for $H=0$. Since isotropic exchange is 

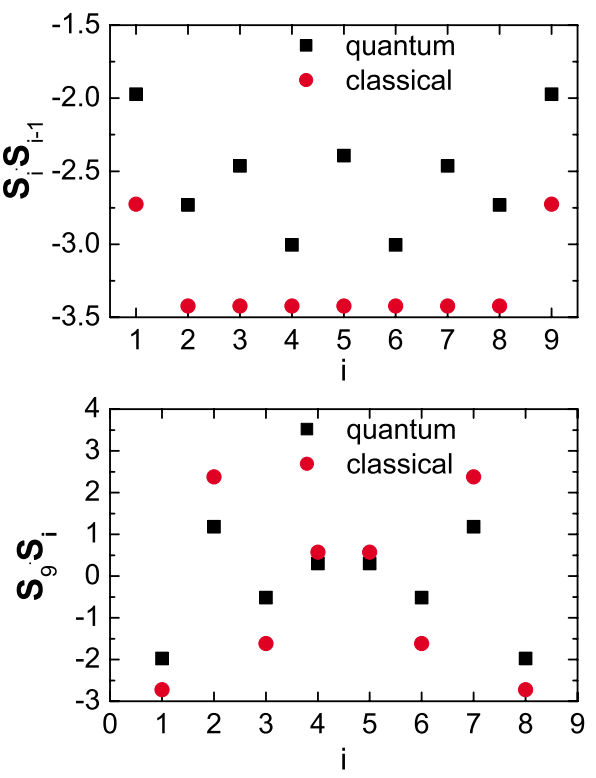

FIG. 7. (Color online) Quantum and classical static two-site correlations resulting from the exchange part of Eq. (1).

the dominant interaction we focus on the ground state of Eq. (1), neglecting crystal-field and DM interactions, which do not qualitatively affect the ground-state properties. In order to investigate the structure of the ground state and, in particular, how this is affected by frustration, we have used the following coupling scheme $\mid S_{18}, S_{189}, S_{27}, S_{36}, S_{2736}, S_{45}$, $\left.S_{273645}, S_{\mathrm{T}}\right\rangle,{ }^{26}$ where $S_{i j k} \ldots$ are quantum numbers for the modulus of $\mathbf{S}_{i}+\mathbf{S}_{j}+\mathbf{S}_{k}+\cdots$. The Ni ion is assumed to be in site 9. We have found that the $S_{\mathrm{T}}=0$ ground state (and the lowest multiplet for each value of $S_{\mathrm{T}}$ up to $S_{\mathrm{T}}=11$ ) is formed by states with $S_{18}=3$ and $S_{189}=2$ for about the $95 \%$. This indicates that the $\mathrm{Cr}$ spins on sites 1 and 8 are practically locked antiparallel to the $\mathrm{Ni}$ spin, and parallel to each other. This originates from the much larger value of $J_{\mathrm{Cr}-\mathrm{Ni}}=85 \mathrm{~K}$ with respect to $J_{\mathrm{Cr}-\mathrm{Cr}}=14.7 \mathrm{~K}$. Thus these three spins behave as an effective spin-2 degree of freedom antiferromagnetically coupled to the two neighboring $\mathrm{Cr}$ spins. To characterize the magnetic alignment of the spins in the ground state, we have calculated static zero-temperature two-site correlations $\left\langle\mathbf{S}_{i} \cdot \mathbf{S}_{j}\right\rangle$ and the corresponding quantities for the classical Hamiltonian corresponding to Eq. (1). For the latter the spins are treated as classical vectors of length $\sqrt{S_{i}\left(S_{i}+1\right)}$. We focus in particular on correlations of the eight $\mathrm{Cr}$ spins with the $\mathrm{Ni}$ spin, shown in Fig. 7(a), and on nearest-neighbor correlations shown in Fig. 7(b). Correlations $\left\langle\mathbf{S}_{\mathrm{Ni}} \cdot \mathbf{S}_{1}\right\rangle$ and $\left\langle\mathbf{S}_{\mathrm{Ni}} \cdot \mathbf{S}_{8}\right\rangle$ reflect the above-mentioned locking of the three spins in an $S_{\mathrm{T}}=2$ state. The meaning of the remaining $\left\langle\mathbf{S}_{\mathrm{Ni}} \cdot \mathbf{S}_{i}\right\rangle$ correlations can be understood by inspecting the vector structure of the classical ground state (see Fig. 8), which shows a pattern of correlations similar to that of the quantum ground state: classically, the spins are oriented in a noncollinear fashion due to spin frustration. For example, the $\mathrm{Cr}$ spins at sites 4 and 5 are almost lying in a plane perpendicular to the $\mathrm{Ni}$ spin which corresponds to nearly vanishing correlations $\left\langle\mathbf{S}_{\mathrm{Ni}} \cdot \mathbf{S}_{4}\right\rangle$ and $\left\langle\mathbf{S}_{\mathrm{Ni}} \cdot \mathbf{S}_{5}\right\rangle$ as shown in Fig. 7(a).

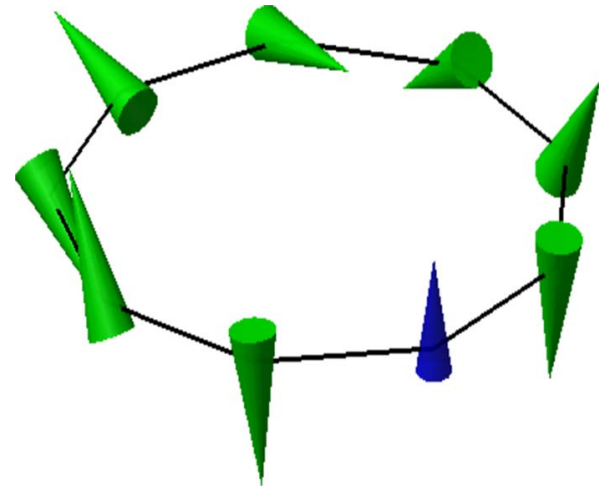

FIG. 8. (Color online) One of the configurations minimizing the energy of the exchange part of the classical version of Eq. (1). The green and blue cones represent $\mathrm{Cr}$ and $\mathrm{Ni}$ spins, respectively.

Quantum mechanically, the $S_{\mathrm{T}}=0$ ground state of $\mathrm{Cr}_{8} \mathrm{Ni}$ approximately resonates among the infinite equivalent classical configurations. Therefore local magnetic moments vanish at low temperature due to quantum fluctuations, which is directly revealed by present NMR measurements.

\section{SUMMARY AND CONCLUSIONS}

We have investigated both experimentally and theoretically the energy structure of low-lying quantum total spin states in an odd-number antiferromagnetic ring $\mathrm{Cr}_{8} \mathrm{Ni}$. Highfield magnetization and specific-heat measurements at low temperatures (below $1 \mathrm{~K}$ ) give clear evidence for a spin singlet ground state at zero magnetic field in agreement with theoretical calculations based on a Hamiltonian including Heisenberg, crystal-field, and Dzyaloshinski-Moriya interactions. We have determined the energy sequence of the excited states, which is also in agreement with the theoretical calculations. The field dependence of the specific heat and the widths of the magnetization steps provide strong evidence of the presence of level anticrossings due to antisymmetric terms in the Hamiltonian. The $\mathrm{Cr}$ spins in the ground state are revealed to have noncollinear orientations due to spin frustration in classical configuration. This is different from the case of nonfrustrated antiferromagnetic ring cluster such as $\mathrm{Cr}_{8} \mathrm{Cd},{ }^{21}$ where $\mathrm{Cr}$ spins align antiferromagnetically in the spin singlet ground state for classical picture. The proton NMR measurements also confirm the singlet ground state of the $\mathrm{Cr}_{8} \mathrm{Ni}$ and reveal that the local spin density is zero in the ground state as a result of quantum fluctuations among frustrated local spin configurations.

\section{ACKNOWLEDGMENTS}

The authors thank A. Lascialfari and G. Amoretti for fruitful discussions. The present work was in part supported by Grant-in-Aid on Priority Areas (Grants No. 451 and No. 473) and for Scientific Research (C) from the Ministry of Education, Culture, Sports, Science, and Technology of Japan, and 
CREST Japan Science and Technology Corporation (JST). The work in Pavia and Parma was supported by NOEMAGMAGNET and COFIN Contract No. 2006029518 (IT). Ames Laboratory is operated for U.S. Department of Energy by Iowa State University under Contract No. W-7405-Eng82. This work at Ames Laboratory was supported by the Director for Energy Research, Office of Basic Energy Sciences.
${ }^{1}$ D. Gatteschi, R. Sessoli, and J. Villain, Molecular Nanomagnets (Oxford University Press, New York, 2006).

${ }^{2}$ L. Thomas, F. Lionti, R. Ballou, D. Gatteschi, R. Sessoli, and B. Barbara, Nature (London) 383, 145 (1996); J. R. Friedman, M. P. Sarachik, J. Tejada, and R. Ziolo, Phys. Rev. Lett. 76, 3830 (1996).

${ }^{3}$ C. Sangregorio, T. Ohm, C. Paulsen, R. Sessoli, and D. Gatteschi, Phys. Rev. Lett. 78, 4645 (1997).

${ }^{4}$ A. Caneschi, A. Cornia, A. C. Fabretti, S. Foner, D. Gatteschi, R. Grandi, and L. Schenetti, Chem.-Eur. J. 2, 1379 (1996)

${ }^{5}$ K. L. Taft, C. D. Delfs, G. C. Papefthymiou, S. Foner, D. Gatteschi, and S. J. Lippard, J. Am. Chem. Soc. 116, 823 (1994).

${ }^{6}$ A. Caneschi, A. Cornia, A. C. Fabretti, and D. Gatteschi, Angew. Chem., Int. Ed. 38, 1295 (1999).

${ }^{7}$ S. P. Watton, P. Fuhrmann, L. E. Pence, A. Caneschi, A. Cornia, G. L. Abbati, and S. J. Lippard, Angew. Chem., Int. Ed. Engl. 36, 2774 (1997).

${ }^{8}$ J. van Slageren, R. Sessoli, D. Gatteschi, A. A. Smith, M. Helliwell, R. E. P. Winpenny, A. Cornia, A. L. Barra, A. G. M. Jansen, E. Rentschler, and G. A. Timco, Chem. Eur. J. 8, 277 (2002).

${ }^{9}$ R. H. Laye, M. Murrie, S. Ochsenbein, A. R. Bell, S. J. Teat, J. Raftery, H. U. Gudel, and E. J. L. McInnes, Chem. Eur. J. 9, 6215 (2003).

${ }^{10}$ G. A. Ardizzoia, M. A. Angaroni, G. LaMonica, F. Cariati, M. Moret, and N. Masciocchi, J. Chem. Soc., Chem. Commun. 15, 1021 (1990).

${ }^{11}$ J. Schnack and M. Luban, Phys. Rev. B 63, 014418 (2000).

${ }^{12}$ D. Gatteschi, L. Pardi, A. L. Barra, A. Muller, and J. Döring, Nature (London) 354, 463 (1991); Y. Furukawa, Y. Nishisaka, K. I. Kumagai, P. Kogerler, and F. Borsa, Phys. Rev. B 75, 220402(R) (2007).

${ }^{13}$ U. Kortz, N. K. Al-Kassem, M. G. Savelieff, N. A. Al Kadi, and M. Sadakane, Inorg. Chem. 40, 4742 (2001).

${ }^{14}$ O. Cador, D. Gatteschi, R. Sessoli, F. K. Larsen, J. Overgaad, A.-L. Barra, S. J. Teat, G. A. Timco, and R. E. P. Winpenny, Angew. Chem., Int. Ed. 43, 5196 (2004); O. Cador, D. Gattes- chi, R. Sessoli, A.-L. Barra, G. A. Timco, and R. E. P. Winpenny, J. Magn. Magn. Mater. 290-291, 55 (2005).

${ }^{15}$ E. J. L. McInnes, S. Piligkos, G. A. Timco, and R. E. P. Winpenny, Coord. Chem. Rev. 249, 2577 (2005).

${ }^{16}$ H. C. Yao, J. J. Wang, Y. S. Ma, O. Waldmann, W. X. Du, Y. Song, Y. Z. Li, L. M. Zheng, S. Decurtins, and X. Q. Xin, Chem. Commun. (Cambridge) (2006) 1745.

${ }^{17}$ S. Carretta, J. van Slageren, T. Guidi, E. Liviotti, C. Mondelli, D. Rovai, A. Cornia, A. L. Dearden, F. Carsughi, M. Affronte, C. D. Frost, R. E. P. Winpenny, D. Gatteschi, G. Amoretti, and R. Caciuffo, Phys. Rev. B 67, 094405 (2003).

${ }^{18}$ M. Affronte, A. Cornia, A. Lascialfari, F. Borsa, D. Gatteschi, J. Hinderer, M. Horvatic, A. G. M. Jansen, and M.-H. Julien, Phys. Rev. Lett. 88, 167201 (2002).

${ }^{19}$ M. Affronte, T. Guidi, R. Caciuffo, S. Carretta, G. Amoretti, J. Hinderer, I. Sheikin, A. G. M. Jansen, A. A. Smith, R. E. P. Winpenny, J. van Slageren, and D. Gatteschi, Phys. Rev. B 68, 104403 (2003)

${ }^{20}$ A. Ghirri, A. Candini, M. Evangelisti, M. Affronte, S. Carretta, P. Santini, G. Amoretti, R. S. G. Davies, G. Timco, and R. E. P. Winpenny, Phys. Rev. B 76, 214405 (2007).

${ }^{21}$ Y. Furukawa, K. Kiuchi, K. I. Kumagai, Y. Ajiro, Y. Narumi, M. Iwaki, K. Kindo, A. Bianchi, S. Carretta, G. A. Timco, and R. E. P. Winpenny, Phys. Rev. B 78, 092402 (2008).

${ }^{22}$ A. Bianchi, S. Carretta, P. Santini, G. Amoretti, T. Guidi, J. Copley, Y. Qiu, G. Timco, and R. Winpenny, Phys. Rev. B (to be published).

${ }^{23}$ S. Carretta, P. Santini, G. Amoretti, M. Affronte, A. Ghirri, I. Sheikin, S. Piligkos, G. Timco, and R. E. P. Winpenny, Phys. Rev. B 72, 060403(R) (2005).

${ }^{24}$ M. Affronte, J. C. Lasjaunias, and A. Cornia, Eur. Phys. J. B 15, 633 (2000).

${ }^{25}$ S. Carretta, P. Santini, G. Amoretti, T. Guidi, J. R. D. Copley, Y. Qiu, R. Caciuffo, G. Timco, and R. E. P. Winpenny, Phys. Rev. Lett. 98, 167401 (2007).

${ }^{26}$ A. Bencini and D. Gatteschi, EPR of Exchange Coupled Systems (Springer-Verlag, Berlin, 1990). 